\title{
Prática de caminhada, atividade física moderada e vigorosa e fatores associados em estudantes do primeiro ano de uma instituição de ensino superior
}

\author{
Practice of walking, moderate and vigorous physical activity and \\ associated factors in first year undergraduate students \\ Gaia Salvador Claumann ${ }^{1 *}$, Érico Felden Pereira ${ }^{1}$, Andreia Pelegrini ${ }^{1}$ \\ ARTIGO ORIGINAL | ORIGINAL ARTICLE
} \begin{abstract}
As mudanças ocorridas com o início da vida universitária podem interferir na prática de atividades físicas de universitários. Como tal, o presente estudo pretendeu investigar a associação entre a prática de caminhada e atividades físicas moderadas e vigorosas com os fatores sociodemográficos e status do peso em estudantes ingressantes no primeiro semestre do primeiro ano de uma universidade pública da região de Florianópolis/SC. Participaram no estudo 198 universitários (86 homens e 112 mulheres). A prática de atividades físicas foi coletada por meio do Questionário Internacional de Atividade Física IPAQ versão curta. Os estudantes das ciências humanas e da educação reportaram praticar maior quantidade de atividade física moderada quando comparados aos estudantes das áreas da saúde e exatas $(p<0.05)$. Ademais, verificou-se que os alunos do sexo masculino, de nível económico alto, da área da saúde e aqueles que não trabalham apresentaram tempo mais elevado de prática de atividade física vigorosa $(p<0.05)$. Verificou-se, por meio da análise de regressão linear, uma associação entre o turno de estudo e a caminhada, e entre as variáveis sexo e área de conhecimento e a atividade física vigorosa. Conclui-se que a associação das variáveis com a prática de atividade física em estudantes universitários do primeiro ano varia conforme o tipo e a intensidade de atividade física

Palavras-chave: atividade física, estudantes universitários, promoção da saúde
\end{abstract}

ABSTRACT

The changes that occur with the beginning of university life may interfere with the practice of physical activities by students. The aim was to investigate the association between the practice of walking, moderate and vigorous physical activities with sociodemographic factors and weight status in freshman students in the first semester of the first year of a public university in Florianopolis/SC. This study assessed198 students (86 men and 112 women). The practice of physical activities was collected with the International Physical Activity Questionnaire - IPAQ, short version. Students of human and educational sciences reported higher amounts of moderate physical activity when compared to health and exact science counterparts $(p<0.05)$. It was verified that male students, from higher economic status, from the health sciences, and full-time students showed higher time of practice of vigorous physical activity $(p<0.05)$. Significant associations were also observed between study period and walking, and between gender, scientific field and vigorous physical activity. It was concluded that the variables associated with the practice of physical activity differ according to the type and intensity of physical activity. Keywords: physical activity, college students, health promotion

Artigo recebido a 28.07.2013; $1^{\text {a }}$ Revisão 04.11.2013; $2^{\text {a }}$ Revisão 14.03.2014; Aceite 03.04.2014

${ }^{1}$ Universidade do Estado de Santa Catarina (UDESC), Florianópolis, Brasil

* Autor correspondente: Centro de Ciências da Saúde e do Esporte, Universidade do Estado de Santa Catarina, Rua Pascoal Simone, 358, CEP: 88080-350, Florianópolis, Brasil; E-mail: gaiasclaumann@hotmail.com 


\section{INTRODUÇÃO}

A prática regular de atividades físicas tem um papel preponderante na promoção de um estilo de vida saudável, o que se evidencia por meio dos inúmeros benefícios físicos, psicológicos e sociais que proporciona em todas as idades (Dias et al., 2008). Apesar destas evidências, a maioria dos jovens não atinge os níveis mínimos recomendados de atividade física (Farias Júnior, 2008) e esta prática tende a diminuir com o avanço da idade, sendo que declínios mais acentuados são observados entre o fim da adolescência ( 15 a 18 anos) e o início da idade adulta (20 a 25 anos) (Marcondelli, Costa, \& Schmitz, 2008). Conforme dados do Censo da Educação Superior realizado pelo Instituto de Estudos e Pesquisas Educacionais Anísio Teixeira (INEP, 2010) a idade mais frequente em que os jovens brasileiros ingressam no ensino superior é 19 anos. Desta forma estudantes universitários, especialmente do $1^{\circ}$ ano, podem constituir um grupo de risco aos níveis insuficientes de atividade física.

Estudos recentes que analisaram o nível de atividade física em universitários corroboram estas informações (Dias et al., 2008; Madureira, Corseuil, Pelegrini, \& Petroski, 2009; Marcondelli et al., 2008). Os resultados encontrados por Sousa (2011), numa revisão sistemática sobre a inatividade física em universitários brasileiros, apontam para elevada prevalência de indivíduos inativos ou insuficientemente ativos fisicamente, o que também tem sido verificado em estudos internacionais (Hawker, 2012; Musharrafieh et al., 2008; Raynor, \& Jankowiak, 2010), independente da área de conhecimento dos estudantes pesquisados e do instrumento utilizado para mensurar a atividade física. Este quadro pode ser explicado devido às situações inerentes ao processo de transição da adolescência para a idade adulta, caracterizado por intensas alterações biológicas e instabilidade psicossocial (Vieira, Priore, Ribeiro, Franceschini, \& Almeida, 2002). Deve-se considerar, também, as mudanças oriundas do ingresso no meio universitário como novas relações sociais e adoção de novos comportamentos. Outro aspeto que pode interferir é o distanciamento da família que normalmente ocorre com o início da vida universitária, quando os jovens experimentam o sentimento de liberdade e independência e passam a ter autonomia sobre seus atos (Vieira et al., 2002).

Neste sentido, as alterações podem implicar diretamente nos níveis de atividade física, tendo em vista que o declínio da prática de atividade física está relacionado a esta independência, pois durante o ensino médio o jovem é obrigado a participar de atividades, ao menos as curriculares, enquanto na universidade a prática passa a ser voluntária (Dias et al., 2008).

Diante do exposto, tem crescido o interesse dos pesquisadores sobre a temática e, consequentemente, o número de publicações (Sousa, 2011). Porém, são escassas informações a respeito da associação dos diferentes tipos e intensidade de atividade física (prática de caminhada, atividade física moderada e vigorosa), variáveis sociodemográficas e status do peso. Neste sentido, é importante verificar se os fatores se associam de maneira semelhante aos tipos e intensidades de atividades físicas. Portanto, o presente estudo tem como objetivo investigar a associação entre a prática de caminhada e atividades físicas moderadas e vigorosas com os fatores sociodemográficos e status do peso em estudantes ingressantes no primeiro semestre de uma universidade pública da região de Florianópolis/SC.

\section{MÉTODo}

Trata-se de um estudo transversal que faz parte do projeto "Nível de atividade física e percepção da imagem corporal em universitários ingressantes", aprovado pelo Comitê de Ética em Pesquisas com Seres Humanos da Universidade do Estado de Santa Catarina (Protocolo n ${ }^{\circ}$ 63452/2012).

\section{Participantes}

A população deste estudo foi constituída por alunos ingressantes no ensino superior no 
primeiro semestre de 2012 em cursos de uma universidade pública cujos centros de ensino estão situados na região de Florianópolis, Santa Catarina. Para o procedimento de seleção amostral, primeiramente, foi realizado um levantamento dos centros situados na região de Florianópolis, dos cursos de graduação e do número de vagas oferecidas para cada curso no vestibular que possibilita acesso à instituição. Os dados foram pesquisados no site da Universidade em abril de 2012 .

$O$ centro em que se encontram os cursos das ciências exatas oferece no primeiro semestre letivo 170 vagas, sendo 80 para o curso de Administração Empresarial (40 para o período vespertino e 40 para o noturno), 50 para o curso de Administração Pública e 40 para o curso de Economia. No centro dos cursos da área da saúde há 90 vagas, destas, 60 para o curso de Educação Física (30 para Bacharelado e 30 para Licenciatura) e 30 para Fisioterapia. $\mathrm{O}$ centro em que são ministrados cursos das ciências humanas e da educação possibilita $\mathrm{O}$ ingresso de 80 alunos, 40 para o curso de História e 40 para Pedagogia. Desta forma, a população da presente pesquisa consistiu em 340 alunos ingressantes na universidade.

Para o cálculo do tamanho amostral foram utilizados os procedimentos sugeridos por Luiz e Magnanini (2000), considerando-se uma prevalência de $85 \%$ de ativos fisicamente (Ferrari et al., 2012), intervalo de confiança de $95 \%$ (IC95\%), erro amostral de 4\%, efeito do desenho de $1 \%$ e acréscimo de $15 \%$ como possível perda amostral. Foi utilizada a amostragem estratificada proporcional por centro de ensino. Com base neste cálculo, estimou-se a necessidade de avaliar 186 universitários, sendo 93 da área das ciências exatas, 49 da área da saúde e 44 das ciências humanas e da educação.

Foram incluídos na presente pesquisa os estudantes universitários presentes em sala de aula no momento da coleta de dados e que aceitaram participar voluntariamente do estudo ao assinar o Termo de Consentimento Livre e Esclarecido.

\section{Instrumentos e Procedimentos}

O nível económico dos indivíduos foi avaliado por meio do questionário da Associação Brasileira de Empresas de Pesquisa (ABEP, 2012), que visa estimar o poder de compra das pessoas e famílias e o grau de instrução do chefe de família, sendo distribuídos em estratos (A1, A2, B1, B2, C, D, E). Os estratos económicos foram agrupados e considerados em nível alto (A1, A2 e B1), médio (B2 e C) e baixo (D e E). Nenhum universitário foi classificado em nível económico baixo, pelo que no presente estudo são apresentadas apenas as categorias alto e médio.

A massa corporal e a estatura foram coletadas por meio de medidas autorreportadas pelos universitários. Segundo a literatura, para esta população, tratando-se de adultos em sua maioria, a correspondência entre medidas referidas e aferidas é satisfatória, de modo que o uso deste método tem sido considerado uma alternativa viável em situações em que não é possível a obtenção direta dessas informações (Coqueiro, Borges, Araújo, Pelegrini, \& Barbosa, 2009), ou quando é preciso tornar o estudo mais rápido e acessível quanto aos custos (Silveira, Araújo, Gigante, Barros, \& Lima, 2005). A classificação do status do peso, a partir do índice de massa corporal (IMC), foi realizada por meio dos pontos de corte estabelecidos pela Organização Mundial de Saúde (WHO, 1998). Em virtude do número baixo de indivíduos classificados com baixo peso $(n=14)$ e obesidade $(n=8)$, no presente estudo, estes foram agrupados e categorizados, respetivamente, em eutróficos (baixo peso + peso ideal) e excesso de peso (sobrepeso + obesidade).

Para avaliação da prática de atividades físicas foi utilizada a versão curta do Questionário Internacional de Atividade Física (IPAQ), sendo a mais indicada para utilização em populações jovens (Guedes, Lopes, \& Guedes, 2005).

\section{Análise Estatística}

$\mathrm{Na}$ análise descritiva das variáveis foram utilizadas médias, desvios padrão e distribuição de frequência. A normalidade dos dados foi 
analisada pelo teste Kolmogorov-Smirnov, e constatou-se que todas as variáveis contínuas não apresentaram distribuição normal. A diferença entre as médias foi verificada pelo teste $U$ de Mann-Whitney e Kruskall-Wallis. O cálculo do tamanho do efeito foi realizado por meio da equação: effect size = valor do teste/ raiz quadrada do $n$ de sujeitos (ou observações) (Field, 2009, pp. 482, 502).

Ademais, recorreu-se a análise de regressão linear simples e multivariada para verificar possíveis associações dos tipos e intensidade de atividade física com as variáveis sociodemográficas e status do peso.

O nível de significância foi estabelecido em $5 \%$ e intervalo de confiança de $95 \%$. Todas as análises foram realizadas no software Statistical Package for Social Sciences (SPSS) versão 20.0.

\section{RESULTADOS}

Participaram do estudo 239 universitários. Destes, 41 foram excluídos devido ao preenchimento incorreto ou incompleto do questionário, totalizando uma amostra final de 198 académicos (86 homens e 112 mulheres) com média de idade de $20.64(\mathrm{DP}=4.56)$ anos, que representam $58.2 \%$ da população estudada.

No presente estudo a maior parte da amostra tem idade inferior a 20 anos $(61.1 \%)$, não trabalha $(55.1 \%)$ e apresenta nível económico alto $(61.1 \%)$. Ademais, a maioria dos académicos estuda no período diurno (73.2\%) e o centro das ciências exatas é onde se concentra o maior número de estudantes (48.5\%).

Observou-se que poucos indivíduos se encontram em condições extremas de status do peso (baixo peso $=7.1 \%$, obesidade $=4.0 \%$ ), sendo a amostra predominantemente eutrófica

Tabela 1

Caracterização geral da amostra de académicos ingressantes numa universidade pública. Florianópolis, SC. (2012)

\begin{tabular}{|c|c|c|c|}
\hline Variáveis & $\begin{array}{c}\text { Masculino }(n=86) \\
\text { M (DP) }\end{array}$ & $\begin{array}{c}\text { Feminino }(n=112) \\
M(D P)\end{array}$ & $p$ \\
\hline Idade (anos) & $21.1(4.2)$ & $20.3(4.8)$ & 0.013 \\
\hline Massa corporal (kg) & $73.9(13.8)$ & $59.2(9.3)$ & $<0.001$ \\
\hline Estatura $(\mathrm{m})$ & $1.8(0.1)$ & $1.6(0.1)$ & $<0.001$ \\
\hline $\operatorname{IMC}\left(\mathrm{kg} / \mathrm{m}^{2}\right)$ & $23.8(4.0)$ & $22.1(3.2)$ & 0.001 \\
\hline Faixa etária, n(\%) & & & 0.001 \\
\hline$\leq 19$ anos & $41(33.9)$ & $80(66.1)$ & \\
\hline$\geq 20$ anos & $45(58.4)$ & $32(41.6)$ & \\
\hline Área de conhecimento, n(\%) & & & 0.109 \\
\hline Ciências da saúde & $23(37.1)$ & $39(62.9)$ & \\
\hline Ciências exatas & $49(51.0)$ & $47(49.0)$ & \\
\hline Ciências humanas e da educação & $14(35.0)$ & $26(65.0)$ & \\
\hline Turno, $\mathrm{n}(\%)$ & & & $<0.001$ \\
\hline Diurno & $51(35.2)$ & $94(64.8)$ & \\
\hline Noturno & $35(66.0)$ & $18(34.0)$ & \\
\hline Trabalho, n(\%) & & & 0.499 \\
\hline $\operatorname{Sim}$ & $41(46.1)$ & $48(53.9)$ & \\
\hline Não & $45(41.3)$ & $64(58.7)$ & \\
\hline Nível económico, n(\%) & & & 0.472 \\
\hline Alto & $55(45.5)$ & $66(54.5)$ & \\
\hline Médio & $31(40.3)$ & $46(59.7)$ & \\
\hline Status do peso, $\mathrm{n}(\%)$ & & & $<0.001$ \\
\hline Eutrófico & $55(36.4)$ & $96(63.6)$ & \\
\hline Excesso de peso & $31(66.0)$ & $16(34.0)$ & \\
\hline
\end{tabular}


(69.2\%). Ainda, verificou-se que os homens apresentaram valores médios estatisticamente superiores para as variáveis idade, massa corporal, estatura e IMC quando comparados às mulheres (Tabela 1).

$\mathrm{Na}$ tabela 2 são apresentados os valores médios da participação em caminhada, atividade física moderada e vigorosa de acordo com o sexo, faixa etária, nível económico, turno de estudo, área de conhecimento, trabalho e status do peso. $\mathrm{Na}$ comparação entre as médias, observou-se diferença na quantidade de atividade física moderada praticada entre as áreas de conhecimento, com valores superiores para a área das ciências humanas e da educação em relação às demais $(p<0.05$; tamanho de efeito $=0.38)$. Os académicos do sexo masculino (tamanho de efeito $=0.15$ ), de nível socioeconómico alto (tamanho de efeito $=0.14$ ), da área da saúde (tamanho de efeito=0.23) e aqueles que não trabalhavam (tamanho de efeito $=0.18)$ apresentaram maior tempo de participação em atividade física vigorosa.

$\mathrm{Na}$ tabela 3, observam-se os fatores associados à prática de caminhada em universitários ingressantes. $\mathrm{Na}$ análise bruta, verificou-se associação apenas com a variável turno de estudo. Os académicos que estudavam no período noturno apresentam mais minutos/semana de caminhada do que aqueles que

Tabela 2

Média semanal de prática de caminhada, atividade física moderada e vigorosa de acordo com o sexo, faixa etária, nível económico, turno, área de conhecimento, trabalho e status do peso de académicos ingressantes. Florianópolis, SC. (2012)

\begin{tabular}{|c|c|c|c|c|c|}
\hline \multirow{2}{*}{ Variáveis } & $\begin{array}{l}\text { Caminhada } \\
(\mathrm{min} / \mathrm{sem})\end{array}$ & & $\begin{array}{l}\text { Moderada } \\
(\mathrm{min} / \mathrm{sem})\end{array}$ & \multirow[b]{2}{*}{$p$} & \multirow{2}{*}{$\begin{array}{c}\begin{array}{c}\text { Vigorosa } \\
(\mathrm{min} / \mathrm{sem})\end{array} \\
\mathrm{M}(\mathrm{DP})\end{array}$} \\
\hline & $\mathrm{M}(\mathrm{DP})$ & $p$ & $\mathrm{M}(\mathrm{DP})$ & & \\
\hline Sexo & & 0.49 & & 0.85 & \\
\hline Masculino & $264.9(294.4)$ & & $278.4(368.4)$ & & $210.6(286.5)^{*}$ \\
\hline Feminino & $230.4(260.0)$ & & $268.2(443.2)$ & & $136.3(204.8)$ \\
\hline Faixa etária & & 0.90 & & 0.66 & \\
\hline$\leq 19$ anos & $233.3(259.0)$ & & $259.9(431.6)$ & & $171.7(231.7)$ \\
\hline$\geq 20$ anos & $264.5(300.0)$ & & $292.6(379.5)$ & & $163.6(267.9)$ \\
\hline Nível económico & & 0.89 & & 0.99 & \\
\hline Médio & $259.7(312.2)$ & & $311.7(503.8)$ & & $132.1(208.8)$ \\
\hline Alto & $236.3(249.9)$ & & $247.8(339.9)$ & & $191.8(264.8)^{*}$ \\
\hline Turno & & 0.25 & & 0.13 & \\
\hline Diurno & $217.6(213.2)$ & & $255.2(354.9)$ & & $151.7(205.0)$ \\
\hline Noturno & $321.6(391.3)$ & & $320.9(537.9)$ & & $214.8(330.8)$ \\
\hline Área de conhecimento & & 0.24 & & 0.02 & \\
\hline Ciências da saúde & $208.7(199.0)$ & & $342.6(400.4)$ & & $254.6(285.2)^{* *}$ \\
\hline Ciências exatas & $214.7(216.5)$ & & $182.8(257.3)$ & & $133.5(179.3)$ \\
\hline $\begin{array}{l}\text { Ciências humanas e da } \\
\text { educação }\end{array}$ & $377.0(429.4)$ & & $379.8(635.3)^{* *}$ & & $119.3(286.8)$ \\
\hline Trabalho & & 0.94 & & 0.90 & \\
\hline Sim & $267.1(315.0)$ & & $291.2(473.1)$ & & $133.5(240.8)$ \\
\hline Não & $227.8(238.1)$ & & $257.4(354.7)$ & & $197.2(247.2)^{*}$ \\
\hline Status do peso & & 0.41 & & 0.69 & \\
\hline Eutrófico & $247.9(270.6)$ & & $258.0(403.7)$ & & $165.2(238.1)$ \\
\hline Excesso de peso & $237.3(292.7)$ & & $319.5(436.4)$ & & $179.5(271.3)$ \\
\hline
\end{tabular}

Nota: n: frequência absoluta; M: média; DP: desvio padrão; * U de Mann-Whitney; ${ }^{* *}$ Kruskall-Wallis. 
Tabela 3

Valores da análise de regressão linear múltipla entre a caminhada e as variáveis sociodemográficas e status do peso de universitários ingressantes. Florianópolis, SC. (2012)

\begin{tabular}{|c|c|c|c|c|c|c|}
\hline Variáveis & $B$ (bruta) & IC95\% & $p$ & $B$ (ajustado) & IC95\% & $p$ \\
\hline \multicolumn{7}{|l|}{ Sexo } \\
\hline Feminino & 1.0 & & & 1.0 & & \\
\hline Masculino & 35.5 & $-43.4 ; 112.4$ & 0.38 & 10.3 & $-74.5 ; 95.0$ & 0.81 \\
\hline \multicolumn{7}{|l|}{ Faixa etária } \\
\hline$\leq 19$ anos & 1.0 & & & 1.0 & & \\
\hline$\geq 20$ anos & 31.2 & $-48.0 ; 110.4$ & 0.44 & 11.2 & $-78.6 ; 101.0$ & 0.81 \\
\hline \multicolumn{7}{|l|}{ Nível económico } \\
\hline Alto & 1.0 & & & 1.0 & & \\
\hline Médio & 40.2 & $-55.9 ; 102.7$ & 0.56 & 10.7 & $-71.2 ; 92.6$ & 0.80 \\
\hline \multicolumn{7}{|l|}{ Turno } \\
\hline Diurno & 1.0 & & & 1.0 & & \\
\hline Noturno & 104.0 & $17.9 ; 190.1$ & 0.02 & 102.1 & $11.2 ; 193.0$ & 0.03 \\
\hline \multicolumn{7}{|c|}{ Área de conhecimento } \\
\hline Outras* & 1.0 & & & 1.0 & & \\
\hline Saúde & -54.4 & $-137.5 ; 28.7$ & 0.20 & -60.3 & $-144.8 ; 24.2$ & 0.16 \\
\hline \multicolumn{7}{|l|}{ Trabalho } \\
\hline Não & 1.0 & & & 1.0 & & \\
\hline $\operatorname{Sim}$ & 39.3 & $-38.2 ; 116.9$ & 0.32 & 24.7 & $-61.0 ; 110.5$ & 0.57 \\
\hline \multicolumn{7}{|l|}{ Status do peso } \\
\hline Eutrófico & 1.0 & & & 1.0 & & \\
\hline Excesso de peso & -10.6 & $-101.5 ; 80.3$ & 0.82 & -47.8 & $-139.4 ; 55.8$ & 0.40 \\
\hline
\end{tabular}

estudavam no período diurno. Esta associação permaneceu na análise ajustada, sendo que os académicos do turno noturno praticam, aproximadamente, 102 minutos a mais de caminhada por semana do que seus pares.

$\mathrm{Na}$ tabela 4, observam-se os fatores associados à prática de atividade física moderada em universitários ingressantes. Tanto na análise bruta quanto na ajustada, nenhuma associação foi verificada entre a prática de atividade física moderada e as variáveis independentes $(p>0.05)$.

$\mathrm{Na}$ tabela 5, observam-se os fatores associados à prática de atividade física vigorosa em universitários ingressantes. $\mathrm{Na}$ análise bruta, as variáveis sexo e área de conhecimento esti- veram associadas ao desfecho, sendo que os académicos do sexo masculino e da área da saúde praticam quantidade superior de minutos por semana de atividades físicas vigorosas. $\mathrm{Na}$ análise ajustada, os académicos da área da saúde apresentaram, aproximadamente, 128.5 minutos/semana a mais de atividade física vigorosa do que os das outras áreas (exatas, humanas e da educação).

\section{DISCUSSÃO}

Muitos estudos que investigaram o nível de atividade física utilizaram o IPAQ versão curta, e apresentaram como objetivo identificar a prevalência de indivíduos ativos e inativos fisicamente e os fatores associados (Maglione, 
Tabela 4

Valores da análise de regressão linear múltipla entre a atividade física moderada e as variáveis sociodemográficas e status do peso de universitários ingressantes. Florianópolis, SC. (2012)

\begin{tabular}{|c|c|c|c|c|c|c|}
\hline Variáveis & $B$ (bruta) & IC95\% & $p$ & $B$ (ajustado) & IC95\% & $p$ \\
\hline \multicolumn{7}{|l|}{ Sexo } \\
\hline Feminino & 1.0 & & & 1.0 & & \\
\hline Masculino & 10.3 & $-106.4 ; 26.9$ & 0.86 & -9.6 & $-137.3 ; 118.0$ & 0.88 \\
\hline \multicolumn{7}{|l|}{ Faixa etária } \\
\hline$\leq 19$ anos & 1.0 & & & 1.0 & & \\
\hline$\geq 20$ anos & 32.7 & $-85.8 ; 151.2$ & 0.59 & 3.1 & $-132.0 ; 138.2$ & 0.96 \\
\hline \multicolumn{7}{|l|}{ Nível económico } \\
\hline Alto & 1.0 & & & 1.0 & & \\
\hline Médio & 63.9 & $-54.3 ; 182.2$ & 0.29 & 64.8 & $-58.5 ; 188.0$ & 0.30 \\
\hline \multicolumn{7}{|l|}{ Turno } \\
\hline Diurno & 1.0 & & & 1.0 & & \\
\hline Noturno & 64.9 & $-65.3 ; 195.2$ & 0.33 & 52.4 & $-84.4 ; 189.2$ & 0.45 \\
\hline \multicolumn{7}{|c|}{ Área de conhecimento } \\
\hline Outras* & 1.0 & & & 1.0 & & \\
\hline Saúde & 101.8 & $-22.0 ; 225.7$ & 0.11 & 113.9 & $-13.3 ; 241.1$ & 0.08 \\
\hline \multicolumn{7}{|l|}{ Trabalho } \\
\hline Não & 1.0 & & & 1.0 & & \\
\hline Sim & 33.8 & $-82.3 ; 149.9$ & 0.57 & 12.2 & $-116.8 ; 141.2$ & 0.85 \\
\hline \multicolumn{7}{|l|}{ Status do peso } \\
\hline Eutrófico & 1.0 & & & 1.0 & & \\
\hline Excesso de peso & 61.4 & $-74.2 ; 197.0$ & 0.37 & 83.3 & $-63.7 ; 230.2$ & 0.27 \\
\hline
\end{tabular}

\& Hayman, 2009; Pelegrini, \& Petroski, 2009; Shuval, Weissblueth, Brezis, Araida, \& DiPietro, 2009; Souza, 2011; Zanchetta et al., 2010). $O$ presente estudo se diferencia ao investigar de maneira isolada a prática de diferentes tipos e intensidades de atividade física entre académicos ingressantes no ensino superior.

Os resultados deste apontam diferenças do tipo e intensidade de atividade física em relação às variáveis sociodemográficas. Os académicos das ciências humanas e da educação apresentaram maior quantidade de prática de atividade física moderada (379.8 $\mathrm{min} / \mathrm{sem}$ ), enquanto os universitários da área da saúde revelaram prática superior de atividade física vigorosa $(254.6 \mathrm{~min} / \mathrm{sem})$. Estes resultados podem ser justificados, em parte, pelo facto de que os universitários das áreas humanas e da educação apresentam faixa etária mais elevada (60\% com idade superior a 20 anos), a maioria trabalha $(67.5 \%)$ e apresentam o menor nível económico (60\% nível médio). Em contrapartida, comparando com as demais áreas de conhecimento, os académicos da área da saúde (Educação Física e Fisioterapia) apresentaram maior proporção no grupo etário inferior a 20 anos $(66.1 \%)$ e a maioria não trabalha (61.3\%). Em relação à caminhada, estudar no turno noturno esteve associado à maior prática de atividades desse tipo. Não foram encontrados na literatura estudos que pudessem confirmar ou refutar essa informação. 
Tabela 5

Valores da análise de regressão linear múltipla entre a atividade física vigorosa e as variáveis sociodemográficas e status do peso de universitários ingressantes. Florianópolis, SC. (2012)

\begin{tabular}{|c|c|c|c|c|c|c|}
\hline Variáveis & $B$ (bruta) & IC95\% & $p$ & $B$ (ajustado) & IC95\% & $p$ \\
\hline \multicolumn{7}{|l|}{ Sexo } \\
\hline Feminino & 1.0 & & & 1.0 & & \\
\hline Masculino & 74.3 & $5.4 ; 143.2$ & 0.04 & 69.9 & $-3.0 ; 142.9$ & 0.06 \\
\hline \multicolumn{7}{|l|}{ Faixa etária } \\
\hline$\leq 19$ anos & 1.0 & & & 1.0 & & \\
\hline$\geq 20$ anos & -8.1 & $-78.9 ; 62.8$ & 0.82 & 1.9 & $-75.3 ; 79.1$ & 0.96 \\
\hline \multicolumn{7}{|l|}{ Nível económico } \\
\hline Alto & 1.0 & & & 1.0 & & \\
\hline Médio & -59.7 & $-130.0 ; 10.6$ & 0.09 & -43.9 & $-114.3 ; 26.6$ & 0.22 \\
\hline \multicolumn{7}{|l|}{ Turno } \\
\hline Diurno & 1.0 & & & 1.0 & & \\
\hline Noturno & 63.2 & $-14.3 ; 140.6$ & 0.11 & 42.4 & $-35.8 ; 120.6$ & 0.29 \\
\hline \multicolumn{7}{|c|}{ Área de conhecimento } \\
\hline Outras* & 1.0 & & & 1.0 & & \\
\hline Saúde & 125.3 & $52.9 ; 197.6$ & $<0.01$ & 128.5 & $55.8 ; 201.2$ & $<0.01$ \\
\hline \multicolumn{7}{|l|}{ Trabalho } \\
\hline Não & 1.0 & & & 1.0 & & \\
\hline Sim & -63.7 & $-132.6 ; 5.1$ & 0.07 & -54.7 & $-128.4 ; 19.0$ & 0.15 \\
\hline \multicolumn{7}{|l|}{ Status do peso } \\
\hline Eutrófico & 1.0 & & & 1.0 & & \\
\hline Excesso de peso & 14.3 & $-66.8 ; 95.4$ & 0.72 & 19.6 & $-64.4 ; 103.6$ & 0.65 \\
\hline
\end{tabular}

A análise de regressão linear simples revelou associação entre a área de conhecimento e a prática de atividades físicas vigorosas, sendo que os académicos das ciências da saúde praticam, aproximadamente, 128.5 minutos a mais por semana de atividades dessa intensidade do que os das demais áreas. Ainda, entre os estudantes da área da saúde, os da Educação Física (Bacharelado e Licenciatura) são os que apresentam maior prática em atividades mais intensas (dados não apresentados). Esses resultados corroboram os estudos de Mielke et al. (2010), Fontes e Vianna (2009) e Marcondelli et al. (2008). Cabe destacar que no presente estudo não foi investigada a existência de atletas entre os universitários, o que possibili- taria um maior entendimento em casos de valores de prática de atividade física, especialmente vigorosa, muito superiores. Supondo que frequentemente atletas ingressam em cursos da área da saúde, caso fossem alvo de investigação, seria possível justificar maior quantidade de atividade física nessa área. Ainda, os cursos de Educação Física possuem no seu plano curricular, já na primeira fase, disciplinas práticas, o que pode ter contribuído para elevar os níveis de atividade física desses. Entre os demais cursos, apenas o de História apresenta alguma prática através de disciplina obrigatória, a Educação Física Curricular; para os outros não existem iniciativas relacionadas à atividade física durante a graduação. Outra 
possível explicação para a maior prática de atividades físicas entre os académicos da área da saúde é a proximidade com os projetos de extensão que se concentram neste centro de ensino. Destaca-se que as instalações do centro das ciências da saúde se encontram afastadas dos demais centros, o que pode dificultar o conhecimento e a participação dos estudantes de outras áreas em práticas regulares de atividade física.

Ademais, entre os indivíduos que não trabalham e apresentam nível económico alto foi observado maior tempo despendido em atividades físicas vigorosas. Deve-se considerar que a média de jornada de trabalho entre os universitários que exercem alguma atividade deste tipo é de 27.4 horas/semana (dados não apresentados), pelo que tal situação somada ao tempo em sala de aula e às demais obrigações académicas pode resultar numa rotina exaustiva, fazendo com que os estudantes, possivelmente, se afastem de atividades físicas que exijam maior esforço e comprometimento, optando, por vezes, por atividades mais livres e tranquilas. O fato de possuir melhor condição económica pode estar relacionado com a possibilidade de se envolver em atividades orientadas e de treino desportivo que apresentam algum custo para participação, como em clubes e academias.

Parece existir consenso na literatura de que os indivíduos do sexo masculino apresentam níveis totais de atividade física superiores aos seus pares do sexo feminino (Farias Júnior, 2008; Maglione \& Hayman, 2009; Marcondelli et al., 2008; Mielke et al., 2010; Papathanasiou et al., 2012; Zanchetta et al., 2010). Os rapazes do presente estudo apresentaram maior envolvimento em atividades físicas vigorosas do que as raparigas, o que corrobora resultados de estudos que encontraram associação entre sexo e intensidade da atividade física (Miller, Staten, Rayens, \& Noland, 2005, Papathanasiou et al., 2012). Verificou-se por meio da análise de regressão linear bruta que eles praticam, aproximadamente, 128 minutos a mais por semana de atividades físicas vigorosas do que elas. Esta associação não se manteve na análise ajustada, havendo, porém, uma tendência de associação $(p=0.06)$. A relação existente entre sexo e intensidade da atividade física pode estar relacionada às diferentes razões de rapazes e raparigas para prática (Mielke et al., 2010). Enquanto eles associam atividade física ao prazer e procuram mais frequentemente práticas desportivas e atividades em grupo, como futebol e basquetebol, elas preferem atividades individuais como caminhada, e preocupam-se por motivos estéticos e de saúde (Monteiro et al., 2003).

Existem evidências de que atividades mais intensas são as que apresentam maiores benefícios para a saúde (Swain \& Franklin, 2005). Entretanto, alguns estudos apontam que, para mulheres, os benefícios relacionados à prática de atividades físicas estão presentes mesmo em intensidades leve e moderada (Pitanga, Lessa, Pitanga, \& Costa, 2011; Pitanga, Pitanga, Beck, Gabriel, \& Moreira, 2012), cabendo, porém, destacar que essas investigações foram conduzidas em amostras com características diferentes das do presente estudo.

Uma das limitações do presente estudo está relacionada à fragilidade e inconsistência do instrumento para verificar a prática de atividade física - IPAQ o qual não permite uma avaliação precisa do tempo despendido em diferentes atividades, além de se mostrar confuso quanto à sua interpretação para os académicos. Outra limitação se refere à utilização de medidas de massa corporal e estatura autorreportadas, que podem ser sub ou superestimadas por alguns académicos, e estes podem, até mesmo, não conhecer suas medidas atuais. Apesar disso, esta forma de obtenção dos dados é considerada válida para a população e tem sido utilizada em estudos prévios (Madureira et al., 2009, Martins et al., 2012).

\section{CONCLUSÕES}

Os resultados encontrados no presente estudo possibilitam concluir, como era esperado, que existem fatores específicos que se associam à prática de atividades físicas de tipos 
e intensidades diferentes em académicos de diferentes áreas de conhecimento. Estudar no turno noturno esteve associado à maior prática de caminhada, enquanto maiores quantidades de atividade física vigorosa estiveram associadas ao sexo masculino e a estudantes da área da saúde. Estas informações são importantes e necessárias para o planeamento e intervenções adequadas a fim de inserir indivíduos em práticas de atividades físicas ou melhorar os níveis atuais de prática entre os diferentes tipos e intensidades de atividades físicas.

Sugere-se que pesquisas futuras investiguem os interesses e motivos, além das barreiras, que levam jovens académicos a praticar ou não atividades físicas, a fim de que novos espaços e projetos sejam planeados para atender a um número maior de indivíduos e às suas diferentes necessidades, reduzindo, assim, a quantidade de indivíduos com baixos níveis de atividade física. Ainda, é importante que iniciativas já existentes sejam divulgadas de maneira mais ampla e eficaz e que existam menos fatores limitantes para a adesão às mesmas.

\section{Agradecimentos:}

Nada a declarar.

\section{Conflito de Interesses:}

Nada a declarar.

Financiamento:

Nada a declarar.

\section{REFERÊNCIAS}

Associação Brasileira de Estudos Populacionais (2012). Critério de Classificação Econômica Brasil. Disponível em http://www.abep.org

Coqueiro, R. S., Borges, L. J., Araújo, V. C., Pelegrini, A., \& Barbosa, A. R. (2009). Medidas autoreferidas são válidas para avaliação do estado nutricional na população brasileira? Revista Brasileira de Cineantropometria e Desempenho Humano, $11(1), 113-119$.
Dias, C., Corte-Real, N., Corredeira, R., Barreiros, A., Bastos, T., \& Fonseca, A. M. (2008) A prática desportiva dos estudantes universitários e suas relações com as autopercepções físicas, bem-estar subjectivo e felicidade. Estudos de Psicologia, 13(3), 223-232. doi: 10.1590/S1413294X2008000300005

Farias Júnior, J. C. (2008). Associação entre prevalência de inatividade física e indicadores de condição socioeconômica em adolescentes. Revista Brasileira de Medicina do Esporte, 14, 109114. doi: 10.1590/S1517-86922008000200005

Ferrari, E. P., Gordia, A. P., Martins, C. R., Silva, D. A., Quadros, T. M., \& Petroski, E. L. (2012). Insatisfação com a imagem corporal e relação com o nível de atividade física e estado nutricional em universitários. Motricidade, 8(3), 5258. doi: 10.6063/motricidade.8(3).1156

Fontes, A. C. D., \& Vianna, R. P. T. (2009). Prevalência e fatores associados ao baixo nível de atividade física entre estudantes universitários de uma universidade pública da região Nordeste. Revista Brasileira de Epidemiologia, 12(1), 2029. doi: 10.1590/S1415-790X2009000100003

Guedes, D. P., Lopes, C. C., \& Guedes, J. E. R. (2005). Reprodutibilidade e validade do Questionário Internacional de Atividade Física em adolescentes. Revista Brasileira de Medicina do Esporte, 11(2), 151-158. doi: 10.1590/S151786922005000200011

Hawker, C. L. (2012) Physical activity and mental well-being in student nurses. Nurse Education Today, 32(3), 325-331. doi: 10.1016/j.nedt. 2011.07.013

Instituto Nacional de Estudos e Pesquisas Educacionais Anísio Teixeira/INEP (2010). Censo da Educação Superior 2010. Brasília: Autor.

Luiz, R. R., \& Magnanini, M. M. F. (2000). A lógica da determinação do tamanho da amostra em investigações epidemiológicas. Cadernos de Saúde Coletiva, 8(2), 9-28.

Madureira, A. S., Corseuil, H. X., Pelegrini, A., \& Petroski, E. L. (2009). Associação entre estágios de mudança de comportamento relacionados à atividade física e estado nutricional em universitários. Cadernos de Saúde Pública, 25(10), 2139-2146. doi: 10.1590/S0102-311X2009001 000005

Maglione, J. L., \& Hayman, L. L. (2009). Correlates of physical activity in low income college students. Research in Nursing \& Health, 32(6), 634646. doi: 10.1002/nur.20353 
Marcondelli, P., Costa, T. H. M., \& Schmitz, B. A. S. (2008). Nível de atividade física e hábitos alimentares de universitários do $3^{\circ}$ ao $5^{\circ}$ semestre da área da saúde. Revista de Nutrição, 21 (1), 3947. doi: 10.1590/S1415-52732008000100005

Martins, C. R., Gordia, A. P., Silva, D. A. S., Quadros, T. M. B., Ferrari, E. P., Teixeira, D. M., \& Petroski, E. L. (2012). Insatisfação com a imagem corporal e fatores associados em universitários. Estudos de Psicologia, 17(2), 241-246. doi: 10.1590/S1413-294X2012000200007

Mielke, G. I., Ramis, T. R., Habeyche, E. C., Oliz, M. M., Tessmer, M. G. S., Azevedo, M. R., \& Hallal, P. C. (2010). Atividade física e fatores associados em universitários do primeiro ano da Universidade Federal de Pelotas. Revista Brasileira de Atividade Física e Saúde, 15(1), 57-64.

Miller, K., Staten, R. R., Rayens, M. K., \& Noland, M. (2005). Levels and characteristics of physical activity among a college student cohort. American Journal of Health Education, 36(4), 215220. doi: 10.1080/19325037.2005.10608187

Monteiro, C., Conde, W., Matsudo, S., Matsudo, V., Bonseñor, I., \& Lotufo, P. (2003). A descriptive epidemiology of leisure-time physical activity in Brazil, 1996-1997. Revista Panamericana de Salud Publica, 14(4), 246-254.

Musharrafieh, U., Tamim, H., Rahi, A., El-Hajj, M., Al-Sahab, B., El-Asmar, K., \& Tamim, H. (2008). Determinants of university students physical exercise: A study from Lebanon. International Journal of Public Health, 53, 208-213.

Papathanasiou, G., Papandreou, M., Galanos, A., Kortianou, E., Tsepis, E., Kalfakakou, V., Evangelou, A. (2012). Smoking and physical activity interrelations in health science students: Is smoking associated with physical inactivity in young adults? Hellenic Journal of Cardiology, 53(1), 17-25.

Pelegrini, A., \& Petroski, E. L. (2009). Inatividade física e sua associação com estado nutricional, insatisfação com a imagem corporal e comportamentos sedentários em adolescentes de escolas públicas. Revista Paulista de Pediatria, 27, 366373. doi: 10.1590/S0103-05822009000400004

Pitanga, C. P. S., Pitanga, F. J., Beck, C. C., Gabriel, R. E., \& Moreira, M. H. (2012). Nível de atividade física para prevenção do excesso de gor- dura visceral em mulheres pós-menopáusicas: Quanto é necessário? Arquivos Brasileiros de Endocrinologia e Metabologia, 56(6), 358-363. doi: 10.1590/S0004-27302012000600003

Pitanga, F. J. G., Lessa, I., Pitanga, C. P. S., \& Costa, M. C. (2011). Atividade física na prevenção de co-morbidades cardiovasculares em mulheres obesas: Quanto é necessário? Revista Brasileira de Atividade Física e Saúde, 16(4), 334-338.

Raynor, D., \& Jankowiak, N. (2010) Accelerometrydetermined adherence to the 2008 Physical Activity Guidelines for Americans among college students. American Journal of Health Education, $41(6), 353-362$.

Shuval, K., Weissblueth, E., Brezis, M., Araida, A., \& DiPietro, L. (2009) Individual and socioecological correlates of physical activity among Arab and Jewish college students in Israel. Journal of Physical Activity and Health, 6, 306-314.

Silveira, E., Araújo, C., Gigante, P., Barros, A. J., \& Lima, M. S. (2005). Validação do peso e altura referidos para o diagnóstico do estado nutricional em uma população de adultos no Sul do Brasil. Cadernos de Saúde Pública, 21 (1), 235-243. doi: 10.1590/S0102-311X2005000100026

Sousa, T. F. (2011). Inatividade física em universitários brasileiros: Uma revisão sistemática. Revista Brasileira de Ciências da Saúde, 9(29), 47-55. doi: 10.13037/rbcs.vol9n29.1293

Swain, D. P., Franklin, B. A. (2006). Comparison of cardioprotective benefits of vigorous versus moderate intensity aerobic exercise. American Journal of Cardiology, 97(1), 141-147.

Vieira, V. C., Priore, S., Ribeiro, S., Franceschini, S. C. C., \& Almeida, L. P. (2002). Perfil socioeconômico, nutricional e de saúde de adolescentes recém-ingressos em uma universidade pública brasileira. Revista de Nutrição, 15(3), 273-282. doi: 10.1590/S1415-52732002000300003

World Health Organization (1998). Obesity: Preventing and Managing the Global Epidemic. Report of a WHO Consultation on Obesity. Geneva: Autor.

Zanchetta, L. M., Barros, M. B. A., César, C. L. G., Carandina, L., Goldbaum, M., \& Alvez, M. C. (2010). Inatividade física e fatores associados em adultos, São Paulo, Brasil. Revista Brasileira de Epidemiologia, 13(3), 387-399. doi: 10.1590/S1415-790X2010000300003 